\title{
The dynamics of phase changes in molecular clusters
}

\author{
L. S. Bartell \\ Department of Chemistry, University of Michigan, Ann Arbor, MI 48109, USA
}

Received 16 September 1992

\begin{abstract}
The significant advantages offered by systems of molecular clusters in the study of homogeneous nucleation are discussed. Determinations of nucleation rates in clusters can be followed experimentally in supersonic jets or computationally in molecular dynamics simulations. Extraordinarily high rates may be encountered, both in freezing and in solid-state transitions. From such information can be inferred the interfacial free energies, $\sigma_{\mathrm{s}}$ and $\sigma_{\mathrm{SS}}$, mechanisms of solid-state transitions. and an explanation of why certain crystalline phases not found in bulk systems can be seen in large molecular clusters.
\end{abstract}

PACS: $36.40 ; 61.14 . F ; 64.60 . Q ; 82.20 . M$

\section{Introduction}

Molecular clusters are proving to be versatile subjects of inquiry in research on condensed matter. In their equilibrium properties they are providing new insights into the crystal chemistry of small, symmetrical molecules. They display phases not seen in conventional crystallographic studies for reasons to be discussed presently. Of perhaps greater significance than their equilibrium structural properties, however, are their dynamic properties, for these show promise in resolving long-standing questions. In the present paper we shall focus upon the dynamics of phase change, paying special attention to the rate at which nucleation occurs. In our laboratory two complementary techniques are applied in the kinetic studies, and both avoid certain formidable troubles which have hindered prior research on nucleation rates. One technique is the probing of clusters by electron diffraction (ED) after they are formed by condensation of vapor in supersonic flow. The other is the numerical simulation of molecular behavior in clusters by molecular dynamics (MD) computations.

By their nature, the techniques are applicable only to rather fast transformations. The very short natural timescale of each together with the extremely small cluster volumes that are subjected to examination restrict the nucleation rates which can be followed with current technology to the range of $10^{27}$ to perhaps $10^{38}$ nuclei per cubic meter per second, with MD rates typically several million-fold faster than those from supersonic jets. Although such rates are astronomical in comparison with those studied in the past by more conventional methods, they do, in fact, occur in real systems of interest. This, in itself, is noteworthy and warrants further pursuit. They have also provided the first examples known to us of rates of nucleation in solid-state phase changes in pure, one-component systems $[1,2]$.

Nucleation plays a crucially important role in many phenomena in science and technology, yet it is poorly understood. The present approach is providing clues useful in its elucidation. We seek to determine the rate of nucleation and its temperature dependence, and to augment such kinetic data, whether from experiment or from simulation, with MD accounts of the molecular behavior during the transitions. Rates of nucleation, interpreted in terms of the classical theory of nucleation, can yield interfacial free energies for boundaries between condensed phases, one or both of which may be solid. Such information is extremely difficult to determine by other methods. Kinetic information also helps establish the mechanism of certain phase changes. In addition, it can explain the occurrence of phases observed in large clusters but not in the bulk. Results can even provide significant tests of nucleation theory, itself. Illustrations of these various applications of the ED and MD techniques will be reviewed in the following sections.

\section{Nucleation rates vs growth rates}

What are observed in individual diffraction experiments are the cluster diameters and the fraction of clusters in the initial and final phases. By following the time-dependence of this fraction, a rate of transformation per unit volume can be calculated. Whether this rate corresponds to the rate of appearance of critical nuclei (which then rapidly transform entire clusters) or to the rate with which the growing phase 
propagates (in already nucleated clusters), cannot be decided by the diffraction results. It is necessary to estimate the rate of propagation of the new phase from other considerations. In most of the transitions monitored to date it has been possible to apply the Wilson-Frenkel theory of diffusionlimited growth rates [3] or to follow the growth rate directly in MD simulations. In all cases so far examined, results have indicated that it is the nucleation rate, not the growth rate, that is rate-limiting, and the distinction has been by many orders of magnitude.

\section{Information derivable from measurements of nucleation rates}

Inference of Interfacial Free Energies: According to the classical theory of homogeneous nucleation the rate of nucleation in freezing or in transitions between two condensed phases can be expressed as [4]

$J=A \exp \left(-\Delta G^{*} / k T\right)$

where $\Delta G^{*}$ is the free energy barrier to the formation of a critical nucleus of the new phase and $A$ is a kinetic prefactor. In those cases where strain energy makes only a minor contribution, $\Delta \mathrm{G}^{*}$ is given by

$\Delta \mathrm{G}^{*}=16 \pi \sigma_{\mathrm{ij}}^{3} /\left(3 \Delta \mathrm{G}_{\mathrm{v}}^{2}\right)$

where $\sigma_{i j}$ is the interfacial free energy between the original and the new phase (averaged over crystal faces) and $\Delta \mathrm{G}_{\mathrm{v}}$ is the Gibbs free energy of transition (in the bulk material) per unit volume. The kinetic prefactor, $A$, is taken to be

$\mathrm{A}=\left(4 \sigma_{\mathrm{ij}} / \mathrm{kT}\right)^{1 / 2} \mathrm{v}_{\mathrm{m}}{ }^{-2 / 3}\left[\mathrm{v}_{\mathrm{o}} \exp (-\mathrm{E} / \mathrm{RT})\right]$

in which $v_{m}$ is the molecular volume, and the bracketed term expresses the frequency with which molecules jump from the matrix phase to the nucleus of the new phase. In a process as simple as freezing, then, a single measured rate $\mathrm{J}$ at a known temperature can lead, via (1) - (3), to a value for the interfacial tension $\sigma_{\mathrm{ij}}$ if the jump rate is inferred from the liquid viscosity. Moreover, because of the occurrence of $\sigma_{i j}$ to the third power in the exponent of (1), an uncertainty in J of many orders of magnitude may lead to an uncertainty in $\sigma_{i j}$ of only a few percent. Misgivings about the thermodynamic significance of a quantity derived from a kinetic measurement with the agency of an imperfect nucleation theory are lessened somewhat by this mathematical insensitivity to error. It turns out that, in those few cases where the kinetic value can be compared with a thermodynamic one, the two usually agree to within the estimated uncertainties [5].

Examples of interfacial free energies derived from experimental nucleation rates are those for the methyl chloromethanes, $\quad\left(\mathrm{CH}_{3}\right)_{n} \mathrm{CCl}_{4-n}$. For technical details associated with the analysis of the highly undercooled systems, see references 1 and 6 . From nucleation rates [6] in freezing of the first two members of the series, $\mathrm{n}=0$ and 1 , we have found values for $\sigma_{\mathrm{sl}}$ of 5.46 and $4.78 \mathrm{~mJ} / \mathrm{m}^{2}$, respectively at the evaporative cooling temperature of the clusters. From the phase III to phase IV solid-state transition of tert-butyl chloride $(n=3)$ we obtained the result $\sigma_{\mathrm{ss}}=3.2 \mathrm{~mJ} / \mathrm{m}^{2}$ [1]. These values are much lower than those reported for the most intensively studied systems, the metals, but are consistent with the much weaker cohesive forces involved. This consistency is particularly evident in the conformity of the present interfacial tensions with Turnbull's empirical relation for the liquid-solid interface [7], namely

$\sigma_{\mathrm{sl}}=\mathrm{k}_{\mathrm{T}}\left[\Delta \mathrm{H}_{\text {fus }} /\left(\mathrm{V}^{2 / 3} \mathrm{~N}_{\mathrm{a}}{ }^{1 / 3}\right)\right]$

where $\mathrm{k}_{\mathrm{T}}$ is a proportionality constant between the interfacial free energy and the bracketed term, the enthalpy of fusion per unit area. Turnbull found that $\mathrm{k}_{\mathrm{T}}$ was approximately 0.5 for metals but that it tended to be 0.32 for the few nonmetals and metalloids examined. Our compounds $\mathrm{CCl}_{4}$ and $\mathrm{CH}_{3} \mathrm{CCl}_{3}$ gave values of 0.35 and 0.32 at the temperatures of nucleation, in close accord with Turnbull's relation. More striking is the discovery that, to within the experimental uncertainties in the values determined so far, the same relation applies also to solidsolid interfacial free energies, and with the same value for $\mathrm{k}_{\mathrm{T}}$, if $\sigma_{\mathrm{sl}}$ and $\Delta \mathrm{H}_{\text {fus }}$ in (4) are replaced by $\sigma_{\mathrm{ss}}$ and the solidstate enthalpy of transition.

Inference of Mechanism of Phase Change: An illustration of the distinction between possible pathways that can be made from the nucleation rate in the absence of more detailed structural information is given by the case of $\left(\mathrm{CH}_{3}\right)_{3} \mathrm{CCl}$. After a comparatively short lag time, the transition from phase III to phase IV took place at an undercooling of $27^{\circ}$. Its rate of $5.5 \times 10^{28} \mathrm{~m}^{-3} \mathrm{~s}^{-1}$ at $156 \mathrm{~K}$ together with its brief lag time turned out to be incompatible with any reasonable extrapolation of NMR results for translational jumps of molecules from phase III to the growing nucleus [1]. The only plausible interpretation was to postulate a reorientational jump, undoubtedly about the C$\mathrm{Cl}$ axis, to usher in the new phase. The structure of the colder phase is as yet unknown. Nevertheless, it is known for phase III that the tert-butyl groups are completely disordered about the $\mathrm{C}-\mathrm{Cl}$ axis [8], and the frequency of reorientational jumps about the axis determined from NMR spectra [9] is consistent with our interpretation of the mechanism. Presumably the tert-butyl groups are ordered in the low temperature phase.

An analogous reorientational transition, this time known to be from bcc to monoclinic, had already been deduced for the case of $\mathrm{SF}_{6}$ from crystallographic and $\mathrm{MD}$ evidence [10]. In this example, $1 / 3$ of the molecules in the disordered bcc structure rotate $60^{\circ}$ about a 3 -fold axis to achieve a more compact packing of molecules, and this stabilizes the colder phase despite the loss of entropy. Direct observations were made of the corresponding transition in $\mathrm{SeF}_{6}$ by electron diffraction [1]. They gave a nucleation rate that was inconsistent with translational jumps of molecules but in harmony with rotational reorientation.

\section{Cluster phases not seen in the bulk}

A number of clusters we have examined have exhibited crystalline phases not yet observed in the bulk, either in crystallographic or in thermochemical studies that covered a 
wide range of temperatures. This difference between clusters and the bulk is not due to any intrinsic difference in packing propensities but to kinetic factors. Clusters of polyatomic molecules are very different in this regard from van der Waals clusters of atoms. Even for quasispherical molecules as, for example, $\mathrm{TeF}_{6}$ an assemblage of fewer than 54 molecules will naturally adopt a bulk-like crystal packing in MD simulations whereas solid Ar clusters with fewer than 1000 atoms preferentially arrange into amorphous structures. Molecular clusters in our supersonic jets typically contain $10^{4}$ molecules and possess lattice constants virtually identical to those of the bulk.

What is very different for clusters is the timescale of observations. In simulations, total times of runs seldom exceed 1 ns. Clusters produced in supersonic flow are seldom examined later than 100 to $200 \mu \mathrm{s}$ after the time of their formation. By contrast, bulk matter in conventional crystallographic and thermochemical investigations is allowed to equilibrate for minutes to days. Another distinction is that the cooling rates of clusters may be higher by many orders of magnitude than any carried out on bulk systems.

The greatest number of cases where previously undetected phases have been found in clusters is among the hexafluorides $\left(\mathrm{AF}_{6}\right)$. These molecules, being highly symmetric and quasispherical can pack in a variety of different periodic arrangements $[11,12]$. Nevertheless, hexafluorides of intermediate size ( $\mathrm{A}=\mathrm{Te}, \mathrm{Mo}-\mathrm{Rh}, \mathrm{W}-\mathrm{Pt}$ ) have been reported to adopt only a warm bcc phase and a low temperature orthorhombic phase [11]. Large, cold clusters of these molecules in supersonic jets, however, are readily produced in a monoclinic phase unknown in the bulk, provided that the clusters are condensed under relatively warm conditions of flow, then quickly cooled. On the other hand, if they are nucleated from the vapor, and grown, under colder conditions, they are orthorhombic. An explanation of why the monoclinic phase is formed in supersonic experiments even though it is less stable than the orthorhombic phase is that the transition from bcc to monoclinic is facile, requiring only a reorientation of onethird of the molecules (see previous section) whereas that for bcc to orthorhombic requires a major reorganization of molecules. Therefore, when the bcc phase is cooled rapidly, the transition to monoclinic outstrips that for the orthorhombic phase and the metastable phase formed, being only marginally less stable, persists.

Yet another phase of $\mathrm{MoF}_{6}$ and $\mathrm{WF}_{6}$ has been observed that has not been detected in the bulk. It has tentatively been identified as rhombohedral. It is produced in supersonic flow when clusters are condensed under even colder conditions than those leading to orthorhombic. Crystal packing calculations and MD simulations of very cold clusters suggest that the rhombohedral structure is even more stable and compact than that of the orthorhombic. We conjecture that the reason it was not observed in the bulk even in heat capacity measurements that extended to very low temperatures, is also kinetic. By the time the temperature has dropped to the orthorhombic-torhombohedral transition temperature, the transformation may be too slow to take place at an appreciable rate. When clusters are produced by condensation from the vapor at very low temperatures, however, the compact rhombohedral structure can grow spontaneously, helped, when the cluster is small, by the Laplace pressure exerted in the cluster's interior.

The occurrence of phases in clusters that are not seen in the bulk, then, can happen in two ways. In some systems undergoing rapid cooling, the speed of a transition to a metastable phase gives the stable phase insufficient time to form. In other circumstances, a thermodynamically stable phase can be grown directly from the vapor but may not be able to be formed on an ordinary laboratory timescale by transition from a higher temperature solid phase by cooling.

\section{Violation of Ostwald's step rule}

One-hundred years ago, W. Ostwald postulated that a "step rule," or "law of successive reactions" applied to transitions [13]. According to this rule, a system passing from a less stable form to a more stable form on cooling does not pass directly to the most stable one if there are intermediate forms that would be encountered if true equilibrium were maintained at all stages. Instead, the system changes into the next most stable form, then the next and so, step by step, into the most stable. This rule helped to account for some phenomena observed, and apparent exceptions were rationalized by supposing that the passage through some intermediates was too fast to be detected.

If our proposed explanation of the generation of rhombohedral clusters of $\mathrm{MoF}_{6}$ and $\mathrm{WF}_{6}$ is correct, Ostwald's rule has been violated. The rule would have required bcc and orthorhombic phases to have been visited before the rhombohedral phase. But current evidence indicates that the orthorhombic phase does not quickly transform to rhombohedral. A more securely identified violation is that of producing orthorhombic clusters of the hexafluorides. They only form in flow too cold for the bcc phase to be produced. If the bcc phase is condensed, and the grown clusters are cooled to a low temperature, orthorhombic clusters are never observed to form. Instead, the clusters transform to monoclinic, where they are trapped on the timescale of the experiments. Clearly, in experiments giving orthorhombic clusters, the bcc and monoclinic phases were never formed.

\section{Tests of nucleation theory}

Experimental studies of nucleation rates at high undercoolings are unlikely, by themselves, to provide very stringent tests of nucleation theory. Quite apart from difficulties in extrapolating the thermodynamic properties and molecular jump rates that are needed for analysis, into regions of high undercooling, it is likely that interfacial free energies can always be invoked that will be able to account for the observed rates. In most cases interfacial free energy can be regarded as a free parameter, modest changes of which can change $J(T)$ by ten orders of magnitude. If it turns out that the interfacial free energies which are found necessary to bring nucleation theory into agreement with experiment are physically entirely plausible, that, of course, is a useful corroboration. The handful of results so far observed from research on molecular clusters appear to be 
plausible inasmuch as they are consistent with Turnbull's proposed relation to heat of transition [7]. Adequate studies of the variation of $\mathrm{J}(\mathrm{T})$ and $\sigma_{\mathrm{ij}}$ with temperature for molecular clusters have yet to be carried out. It is believed that $\sigma_{\mathrm{sl}}$ increases with temperature for metals and it is wellknown that $\sigma_{1 \mathrm{v}}$ decreases. It would be of particular interest to learn how $\sigma_{\mathrm{sl}}$ and $\sigma_{\mathrm{ss}}$ respond to changes in temperature in molecular systems.

The commonest criticism of classical nucleation theory is its attribution of bulk-like properties to submicroscopic critical nuclei. In condensation from the vapor, where surface tensions of bulk phases seem not to represent the temperature dependence of nucleation rates very faithfully [14], the critical nuclei are extraordinarily small. They may be no larger than 5 or 10 molecules in many cases. Even if Tolman's size correction is introduced to reduce the discrepancy with experiment, it takes an act of faith to model nuclei that small on the basis of bulk measurements. Critical nuclei are an order of magnitude larger in the supersonic experiments on phase changes in clusters and might conceivably be better represented by bulk-like properties.

One of the most promising approaches for testing nucleation theory in systems of molecular clusters is to carry out MD simulations. Several years ago Swope and Andersen [15] examined nucleation in the freezing of a bulk system of Lennard Jones spheres and found several elements of correspondence with classical nucleation theory. Enormous systems were found necessary ( $>10^{4}$ molecules) if serious artifacts introduced by periodic boundary conditions were to be avoided. In our work we bypass periodic boundary conditions by working with clusters. Although this avoids certain sources of pathology, it does introduce an uncertainty in the effective volume for nucleation. In small clusters the interior molecules are by far the most likely candidates for forming nuclei in cooling runs but molecules in the outermost layer may outnumber them. Be that as it may, we have been able to follow both freezing and solid-state phase transitions in systems of molecular clusters. Of special interest because of the dearth of prior information about processes in the solid state is the rapid bcc to monoclinic transition in several hexafluorides. In such reactions the technique of identifying critical nuclei that was used in the freezing of atomic systems (analyzing Voronoi polyhedra [15]) did not work. What does allow the nuclei to be detected when they are formed and while they grow are the cooperative molecular reorientations that accompany the phase change. These can be seen in the Pawley-Fuchs projections of bond directions [16]. In order of magnitude these nuclei are of the size predicted by nucleation theory. Although a recent MD simulation [17] of phase changes in $\mathrm{CCl}_{4}$ has identified quantitative deficiencies in the capillary model lying at the heart of the classical theory of nucleation, many aspects of the capillary model are at least qualitatively correct. Further research on the dynamics of formation of critical nuclei should provide additional insight into the virtues and limitations of existing nucleation theory.

This research was supported by a grant from the National Science Foundation. Important contributions to the research discussed were made by Dr. T. S. Dibble and Messrs. S. Xu, P. Lennon, J. Hovick, and $\mathrm{K}$. Kinney.

\section{References}

1. Dibble, T. S., Bartell, L. S.: J. Phys. Chem. in press

2. Beck, R. D., Hineman, M. F., Nibler, J. W.: J. Chem. Phys. 92, 7068 (1990) report observing the $\beta$ to $\alpha$ solid-state transitions in clusters of $\mathrm{N}_{2}$ but do not discuss nucleation rates

3. Wilson, H. A.: Phil. Mag 50, 238 (1900); Frenkel, J.: Phys. Z. Sowjetunion 1, 498 (1932)

4. Buckle, E. R.: Proc. R. Soc. London A 261, 189 (1961)

5. Eustathopolous, N.: Int. Met. Rev. 28, 189 (1983)

6. Dibble, T. S., Bartell, L. S.: J. Phys. Chem. 96, 2317 (1992)

7. Turnbull, D.: J. Appl. Phys. 21, 1022 (1950)

8. Schwartz, R.S., Post, B., Fankuchen, I.: J. Am. Chem. Soc. 73, 4490 (1951)

9. Goyal, P. S., et al.: Acta Phys. P. A 46, 399 (1974)

10. Pawley, G. S., Dove, M. T.: Chem. Phys. Lett. 99, 45 (1983)

11. Siegel, S., Northrup, D. A.: Inorg. Chem. 5, 2187 (1987)

12. Taylor, J. C.: Coord. Chem. Rev. 20, 197 (1976)

13. Ostwald, W.: Lehrbuch der Allgemeinen Chem. Vol.II-2, p. 445. Leipzig: Englemann 1893.

14. Hung, C., Krasnopoler, M. J., Katz, J. L.: J. Chem. Phys. 90, 1856 (1989)

15. Swope, W. C., Andersen, H. C.: Phys. Rev. B 41, 7042 (1990)

16. Fuchs, A. H., Pawley, G. S.: J. Phys. France 49, 41 (1988)

17. Bartell, L. S., Chen, J.: J. Phys. Chem. in press 